\title{
Artificial Bee Colony Based Focus Fusion
}

\author{
Veysel Aslantas, Ahmet Nusret Toprak \\ Erciyes University, Computer Engineering Department \\ Kayseri, TURKEY \\ aslantas@erciyes.edu.tr, antoprak@erciyes.edu.tr
}

\begin{abstract}
This paper proposes a pixel based multi-focus image fusion method which consists of two main steps: detection of point spread functions of the source images by using artificial bee colony algorithm and fusion of the source image by making use of estimated point spread functions. The main advantage of the proposed method is it directly fuses the source images without any transformation that modifies the original pixel values. Experimental results demonstrate the superiority of the proposed method in terms of quantitative and visual evolution.
\end{abstract}

Keywords—multi-focus image fusion; artificial bee colony, blur estimation

\section{INTRODUCTION}

Images taken by digital cameras usually suffer from limited depth of field (DoF) problem. Limited DoF disallows the digital cameras to take all-in-focus images. Therefore the obtained image appears partially blurred. Multi-focus image fusion techniques solve this problem by taking multiple images with different focus points and fusing them together to obtain an all-in-focus image [1]. Multi-focus image fusion techniques have been used widely in digital camera and microscopy applications.

The multi-focus image fusion methods generally divided into two groups: spatial domain methods are based on direct fusion of pixels of the input images; transform domain processing methods are based on fusing the transforms of the images [2].

Transform domain based methods consist of three main steps: applying a transform to the each input images, fusing all transforms to produce the fused representation, and at last applying the inverse transform to the fused representation to obtain the final fused image. The most generally used transform based methods can be listed as Laplacian Pyramid (LP) [3], Discrete Wavelet Transform (DWT) [4], and Discrete Cosine Transform (DCT) [5]. However, applying a transform prior to fusion modifies the original pixels of input images. Therefore, brightness and color distortions may occur on the fused image [6].

Different from the transform domain based methods, spatial domain based methods directly fuse the input images by taking their spatial features into account. These methods search for sharp pixels or regions to be transferred in order to construct the fused image. Spatial domain based divided into two main groups: region based and pixel based methods. The former methods such as block selection method [7], Differential Evolution based block selection [8], region selection [9], initially segment the input images by a segmentation algorithm and then produce fused image by selecting the sharper regions.
The latter methods such as spatial frequency based method [10] determine the sharp pixels that form the fused image.

In this paper, a pixel based spatial domain method is proposed. The proposed method has two main steps: estimating the point spread functions (PSF) of input image and fusing input images by using the estimated PSFs. In the multi-focus image fusion applications, a collection of images with different focus points is captured. Each image of this collection includes both blurred and sharp regions. While a particular object in the scene appears in focus in one image, it appears out of focus in others. In other words, both blurred and sharp images of each object in the scene exist in the collection. Blurred image of an object can be defined as the convolution of the same object with a particular PSF [11]. This means if the both blurred and sharp images exist, then the PSF can be calculated. However, there is no prior knowledge about which region of the input images are blurred or sharp. Therefore a novel technique that using $\mathrm{ABC}$ to estimate the PSFs of the input images is proposed in this paper. $\mathrm{ABC}$ is simple yet effective. Therefore it is straightforward to solve optimization problems. $\mathrm{ABC}$ has been also employed to solve several problems in image processing area. A good review can be found in [12]. The proposed method, firstly calculate the PSFs of the input images by using ABC. Then, it detects sharp pixels by making use of estimated PSFs. Finally, it forms the fused image by transferring the detected sharp pixels.

The rest of paper is organized as follows. Section II explains the proposed method in detail. Experimental results are given in Section III. Finally, Section IV concludes the paper.

\section{FOCUS FUSION BASED ON ARTIFICIAL BEE COLONY}

In this section, firstly, the $\mathrm{ABC}$ algorithm is briefly described. Then, the ABC based PSF estimation and focus fusion methods are introduced. 


\section{A. Artificial Bee Colony $(A B C)$ Algorithm}

Artificial Bee Colony (ABC) is a swarm based meta-heuristic global optimization algorithm that was defined by Karaboga [13]. It is motivated by the intelligent foraging behavior of the honey bees. $\mathrm{ABC}$ has only three control parameters: population size, maximum cycle number and limit.

In $\mathrm{ABC}$ algorithm, each possible solution is represented by a food source and the nectar amount of the food sources represent the quality of the corresponding solution. The artificial bee colony consists of three groups of bees: employed bees which are employed at a specific food source, onlooker bees that watch the dance of employed bees to choose a food source, and scout bees that search the environment randomly. Both of the onlookers and the scouts are also called as unemployed foragers. Initially, all food sources are explored by scout bees. Then, the nectar of food sources are exploited by employed and onlooker bees and this cause them to become exhausted. Afterwards, the employed bees whose food source is exhausted become a scout bee. The number of the employed bees is equal to the number of food sources (solutions).

At the first step, the initial population of the $\mathrm{SN}$ food sources is initialized by the scout bees where $\mathrm{SN}$ denotes the number of employed bees. Each food source $x_{\mathrm{m}}(m=1,2, \ldots, S N)$ is a solution vector with $n$ variables $x_{\text {mi }}(i=1,2, \ldots, n)$ which are the optimization parameters. In order to initialize the food sources the following formula is used.

$$
x_{m i}=l_{i}+\operatorname{rand}(0,1) *\left(u_{i}-l_{i}\right)
$$

where $l_{i}$ and $u_{i}$ are the lower and the upper bounds, respectively.

After the initialization, there are three phases of $A B C$ algorithm. First one is employed bees phase. Employed bees search for the new food sources $\left(v_{m}\right)$ that have more nectar, in the neighborhood of the food source $\left(x_{m}\right)$ in their memory. They determine a neighbor food source using the following definition.

$$
v_{m i}=x_{m i}+\phi_{m i}\left(x_{m i}-x_{k i}\right)
$$

where $x_{k}$ is randomly chosen food source, $i$ is a randomly selected parameter, $\phi$ is a random number. After producing the new food source $v_{m}$, its fitness value is calculated and the food source that has the best fitness value is selected between $x_{m}$ and $v_{m}$.

Second phase is the onlooker bees phase. As mentioned above, there are two kind of unemployed bees: onlooker and scout bees. Employed bees share the information about their food source with the onlooker bees. Then onlooker bees choose their food source by considering the probability value that calculated using the fitness value providing by employed bees. The probability value $p_{m}$ is calculated by using the following expression.

$$
p_{m}=\frac{f\left(x_{m}\right)}{\sum_{m=1}^{S N} f\left(x_{m}\right)}
$$

where $f$ is the fitness function.

After food source $\left(x_{m}\right)$ is selected, a neighbor food source $v_{m}$ is found out by using the eq. (2). As in the previous phase, a greedy selection is applied between $x_{m}$ and $v_{m}$.

The last phase is the scout bees phase. Scout bees are the unemployed bees that search their food sources randomly. If an employed bee cannot improve its solution through a predefined number of iterations called limit, it becomes scout bee and its solutions are deserted. Thereafter, converted scout bee start to search for new food source by using (1).

\section{B. ABC Based PSF Estimation}

In this section a novel method that uses $\mathrm{ABC}$ to estimate the PSF function of the multi-focus input image is presented. Each input image of multi-focus collection includes both focused (sharp) and defocused (blurred) regions. For the simplicity, assume that collection contains two input images. Input images $\left(I_{I}\right.$ and $\left.I_{2}\right)$ can be defined by following definitions.

$$
\begin{aligned}
& I_{1}=f_{1}(x, y)+g_{1}(x, y) \\
& I_{2}=f_{2}(x, y)+g_{2}(x, y)
\end{aligned}
$$

where $f(\mathrm{x}, \mathrm{y})$ and $g(\mathrm{x}, \mathrm{y})$ represent the focused and defocused pixels of input images, respectively. Since defocused image of an object is equal to convolution of the focused image of same object with a particular PSF, defocused image $g(\mathrm{x}, \mathrm{y})$ can be written as following equation.

$$
g(x, y)=f(x, y) \otimes h(x, y, \sigma)
$$

where $h(x, y, \sigma)$ is the PSF function. The PSF of an imaging system describes the shape of the blur formed when a point object is imaged. PSF best fit to a 2D Gaussian model [14]:

$$
h(x, y)=\left(1 / 2 \pi \sigma^{2}\right) \exp \left(-\left(x^{2}+y^{2}\right) / 2 \sigma^{2}\right)
$$

where $\sigma$ denotes the spread parameter (SP).

Proposed PSF estimation method employs the ABC in order to estimate the PSFs of the input images. As can be seen from (6), the only parameter of the PSF is spread parameter $(\sigma)$. Thus, SPs of the input images are searched for each input image in the optimization process. In our example, each individuals of ABC consist of two parameters that are the SPs ( $\sigma_{1}$ and $\sigma_{2}$ ) of PSFs of the input images $\left(h_{1}\right.$ and $h_{2}$ ).

Initially, the control parameters of the $\mathrm{ABC}$ maximum cycle number, population size and limit are defined. Then initial population is generated randomly by using (1).

In the each cycle of $\mathrm{ABC}$, for each individual following steps are repeated until stopping criteria is met. First, fitness value of the each individual is calculated. To this end, PSFs are produced by substituting spread parameter of each individual in (6). Artificially blurred images are produced by convolving each input image with the other's PSF. In our example, $I_{1}$ is convolved with $h_{2}$ and $I_{2}$ is convolved with $h_{1}$ to generate artificially blurred images $\hat{I}_{1}$ and $\hat{I}_{2}$, respectively. 


$$
\begin{aligned}
& I_{1}(x, y)=I_{1}(x, y) \otimes h_{2}\left(x, y, \sigma_{1}\right) \\
& I_{2}(x, y)=I_{2}(x, y) \otimes h_{1}\left(x, y, \sigma_{2}\right)
\end{aligned}
$$

Then, difference images $\left(D_{1}\right.$ and $\left.D_{2}\right)$ are obtained by subtracting $I_{1}$ from $\hat{I}_{2}$ and $I_{2}$ from $\hat{I}_{1}$.

$$
\begin{aligned}
& D_{1}=\left|I_{1}-I_{2}\right| \\
& D_{2}=\left|I_{2}-I_{1}\right|
\end{aligned}
$$

If the optimum PSFs are obtained, the dot product of the difference image should be equal to zero. Thus the following definition is used as the fitness function.

In order to generate the next generation of the population, employed bees, onlooker bees and scout bees phases are executed, respectively. If the stopping criterion is not met, the above steps are repeated.

\section{Fusion of Input Images Using Optimal PSFs}

After the estimation of optimal PSFs of the input images, sharp images can be determined. First artificially blurred images $\left(\hat{I}_{1}\right.$ and $\left.\hat{I}_{2}\right)$ are produced by substituting optimally estimated PSFs in (7). Since the PSFs that are used to generate the artificially blurred images are optimal, blurred regions of input images are obtained blurred with same degree in artificially blurred ones. In other words, pixel values of the blurred regions of the input images are equal to values of the corresponding pixels in the artificially blurred ones.

Once the artificially blurred images are produced, difference images $\left(D_{1}\right.$ and $\left.D_{2}\right)$ can be calculated by using (8). Since optimal PSFs are used, blurred regions $(g(x, y))$ of input images $\left(I_{1}\right.$ and $\left.I_{2}\right)$ are canceled out in difference images $\left(D_{1}\right.$ and $D_{2}$ ), respectively. In this manner, each pixel of the difference images is compared as following expression.

$$
I_{F}(x, y)= \begin{cases}I_{1}(x, y) & D_{1}(x, y) \geq D_{2}(x, y) \\ I_{2}(x, y) & D_{1}(x, y)<D_{2}(x, y)\end{cases}
$$

Fused image $\left(I_{F}\right)$ is generated by transferring pixels from input image which corresponding value of difference image is bigger.

\section{EXPERIMENTAL RESULTS}

In this section the visual and quantitative evaluation about fusion performance of the proposed method is reported. The results of the proposed method are compared with three well-known methods: Discrete Wavelet Transform (DWT) [4], Discrete Cosine Transform (DCT) [5] and Pixel based method (PB) [10]. The parameters of the methods are selected as: for DWT filter is "Sym8" and decomposition level is 7; for PB sharpness value is spatial frequency (SF). For the proposed method, as a result of many experiments control parameter of $\mathrm{ABC}$ is selected as: $\mathrm{NP}=16, \mathrm{~L}=20$.

To assess the performance of the fusion methods quantitatively, three objective quality metric: quality of edges $\left(Q_{E}\right)$ [15], mutual information $\left(Q_{M I}\right)$ [16] and structural similarity $\left(Q_{S S I M}\right)$ [17] are used. Experiments are conducted on three different multi-focus image sets: Clock, Matches and Watch. The input images of these sets are illustrated in Fig. 1.
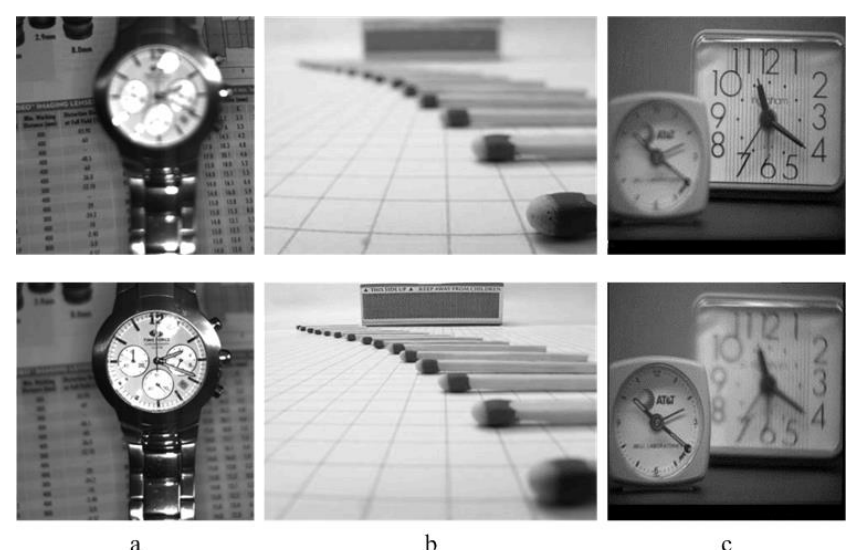

Fig. 1. Input image sets: (a) Watch, (b) Matches, (c) Clock.

The first experiment is carried out on the Watch image set. In this image set there is a watch on a paper. In the first image watch is focused and in the other background is focused. The fusion results and the difference images that are obtained by subtracting fused image from each input image, of the Watch image set is given in Fig. 2. The small parts of the watch make this image set challenging. As can be seen from the Fig. 2. transform domain based methods DWT and DCT produce artifacts around the detail parts of watch. This artifact can be easily seen in difference images of these methods. Although there are also some minor artifacts in the fused image of PB method, it introduces an acceptable result. By contrast, the proposed method does not produce any artifacts and well preserve detail parts of the watch.

The experiments are also performed on the Matches image set. In this image set match sticks are arranged in order and a matchbox is also located at the background of the images. Fused and difference images of the fusion methods are shown in the Fig. 3. As can be observed from the images DCT method produces reasonable result. However, first match is blurred in the fused image. On the other hand, some major artifacts exist in the results of DWT and PB methods. These especially cannot preserve the match heads. On the contrary, proposed method well preserves matches and the matchbox on the fuse image.

The last experiments are conducted on the Clock image set. In this image set there are two clocks on a table. In the each image one of the clocks is focused. The fusion results of the Clock image set is given in Fig. 4. Difference images of DWT reveal that DWT cannot well preserve the details of the clocks and modifies the original pixel values. On the other hand DCT and PB methods introduce blocking artifacts. By contrast, the proposed method can effectively fuse the images and preserves the details. 
The objective performance of the methods is also evaluated. Table 1 gives the objective quality values of the methods in terms of $Q_{E}, Q_{M I}$ and $Q_{S S I M}$ metrics. From the table, it can be seen that the proposed method provides the best fusion results in terms of $Q_{E}, Q_{M I}$ and $Q_{S S I M}$ metrics. For Clock image DCT produce a better $Q_{M I}$ result. However this results does not coincide with the visual result.

TABLE I. QuANTITATIVE Assesment of Fusion Methods

\begin{tabular}{|c|c|c|c|c|c|}
\hline \multirow{2}{*}{ Images } & \multirow{2}{*}{ Metrics } & \multicolumn{4}{|c|}{ Methods } \\
\cline { 2 - 6 } & & DWT & DCT & PB & Proposed \\
\hline \multirow{3}{*}{ Watch } & $\boldsymbol{Q}_{\boldsymbol{E}}$ & 0,6488 & 0,7167 & 0,7217 & $\mathbf{0 , 7 2 2 6}$ \\
\cline { 2 - 6 } & $\boldsymbol{Q}_{M I}$ & 5,6141 & 9,3345 & 9,2185 & $\mathbf{9 , 3 3 9 8}$ \\
\cline { 2 - 6 } & $\boldsymbol{Q}_{\text {SSIM }}$ & 0,9514 & 0,9766 & 0,9755 & $\mathbf{0 , 9 7 7 1}$ \\
\hline \multirow{3}{*}{ Matches } & $\boldsymbol{Q}_{\boldsymbol{E}}$ & 0,7215 & 0,7658 & 0,7826 & $\mathbf{0 , 7 8 9 5}$ \\
\cline { 2 - 6 } & $\boldsymbol{Q}_{M I}$ & 7,6860 & 12,5990 & 12,3110 & $\mathbf{1 2 , 3 9 4 8}$ \\
\cline { 2 - 6 } & $\boldsymbol{Q}_{\text {SSIM }}$ & 0,9810 & 0,9753 & 0,9834 & $\mathbf{0 , 9 8 5 2}$ \\
\hline \multirow{3}{*}{ Clock } & $\boldsymbol{Q}_{\boldsymbol{E}}$ & 0,6710 & 0,7387 & 0,7386 & $\mathbf{0 , 7 3 8 8}$ \\
\cline { 2 - 6 } & $\boldsymbol{Q}_{M I}$ & 5,8839 & $\mathbf{9 , 0 8 0 0}$ & 9,0406 & 9,0413 \\
\cline { 2 - 6 } & $\boldsymbol{Q}_{S S I M}$ & 0,9590 & 0,9806 & 0,9790 & $\mathbf{0 , 9 8 1 2}$ \\
\hline
\end{tabular}

\section{CONCLUSION}

In this paper, an effective multi-focus image fusion method is proposed. Furthermore, a method that estimates the PSFs of the multi-focus input images by using $\mathrm{ABC}$ is also introduced. The proposed multi-focus image fusion method initially estimates the PSFs of the input images. Then each input image is artificially blurred by convolving estimated PSFs. By using these artificially blurred images, sharp pixels of the input images are determined. Eventually, the all-in-focus fused image is generated by gathering the sharp pixels. Experiments show that the proposed method can produce fused images that have high visual quality. Moreover, the effectiveness of the proposed method is demonstrated by quantitative metrics.

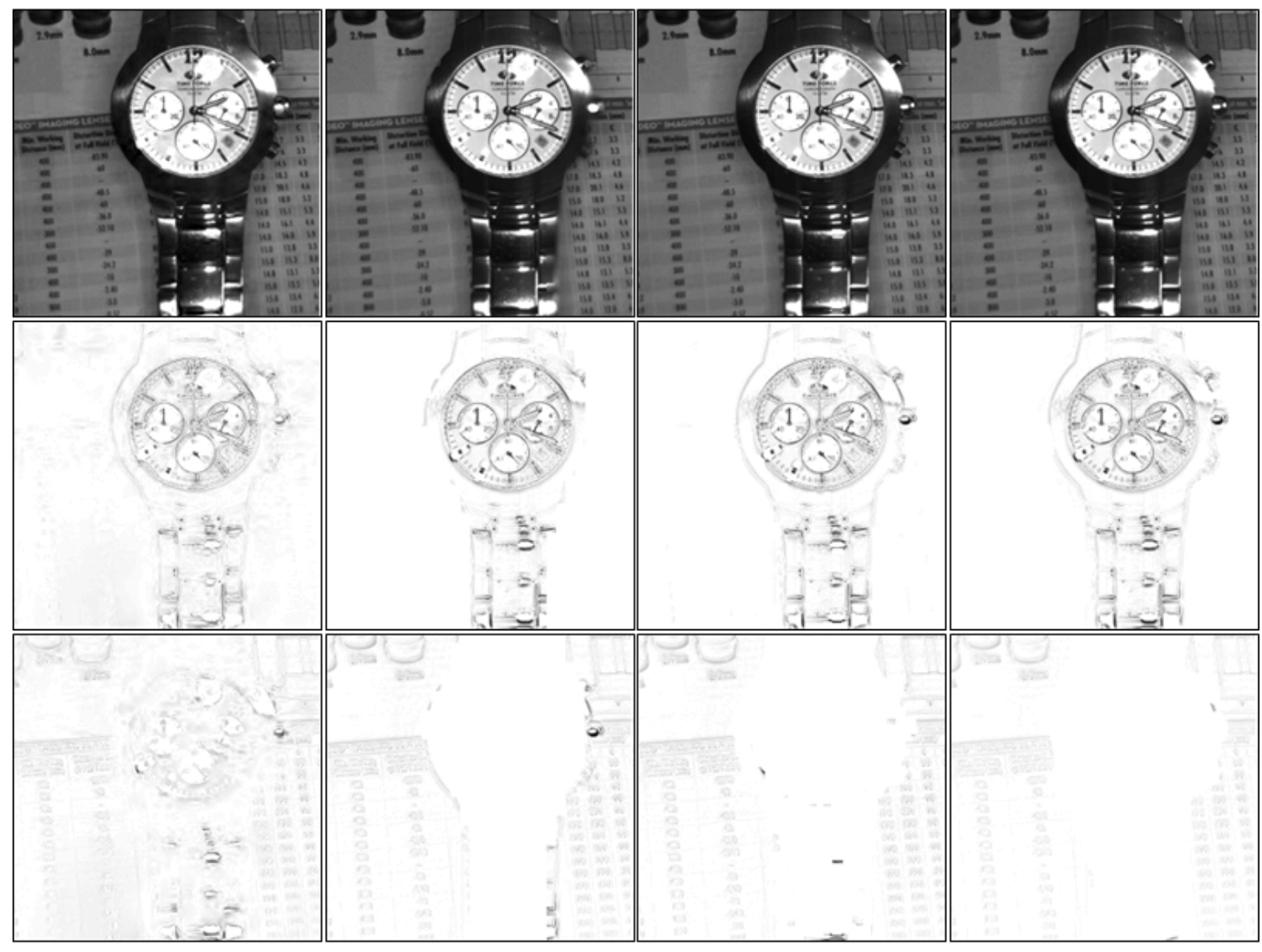

a

b

c

Fig. 2. Fused and difference images of the Watch image set: (a) DWT (b) DCT (c) PB (d) Proposed method. 
ICIT 2015 The $7^{\text {th }}$ International Conference on Information Technology

doi:10.15849/icit.2015.0021 @ ICIT 2015 (http://icit.zuj.edu.jo/ICIT15)

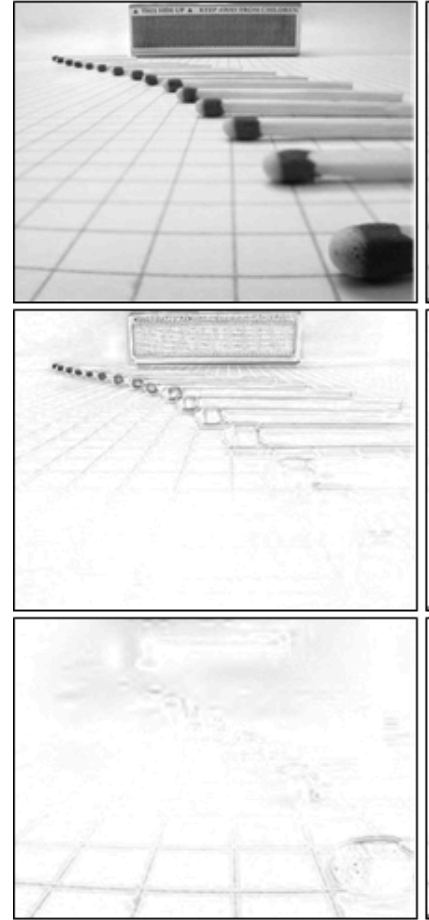

a
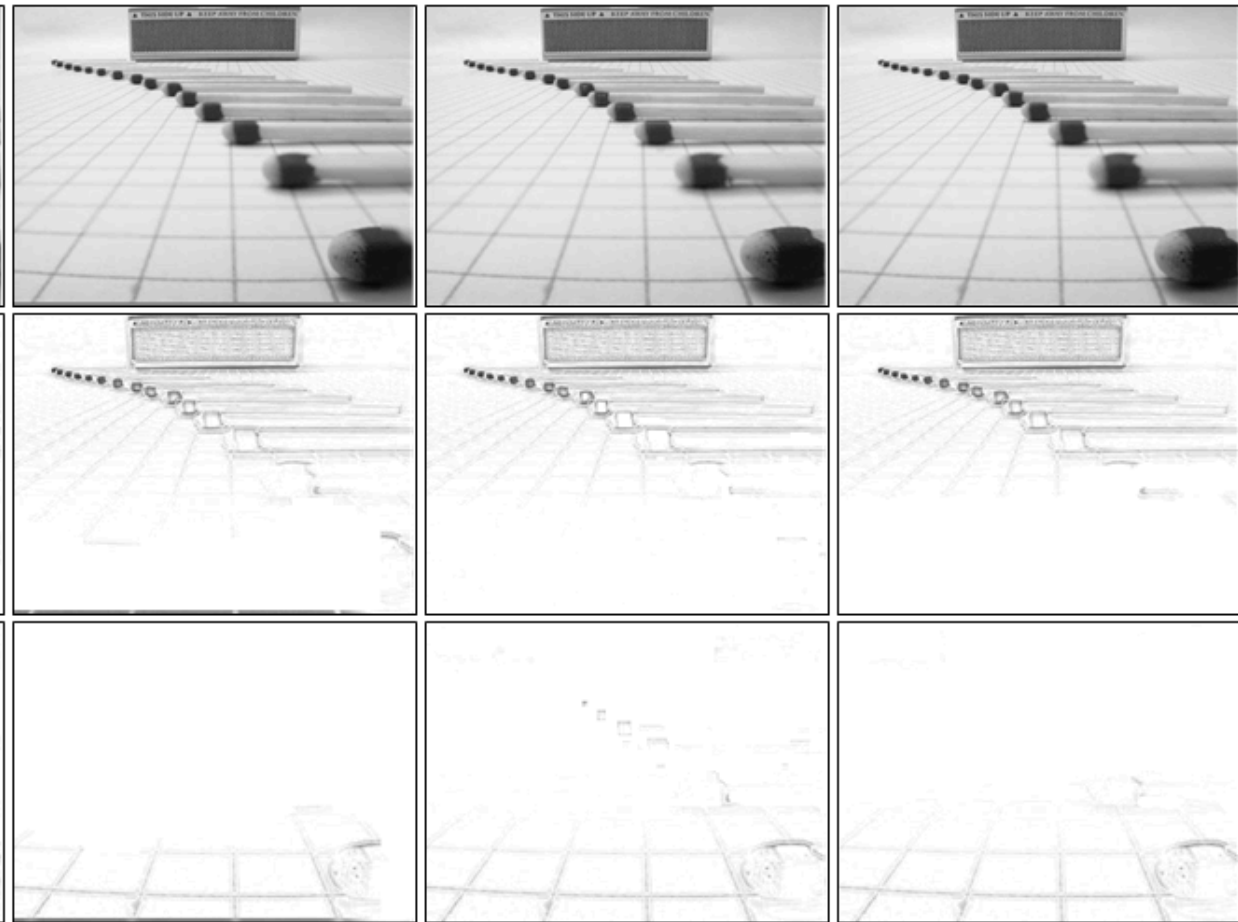

$\mathrm{b}$

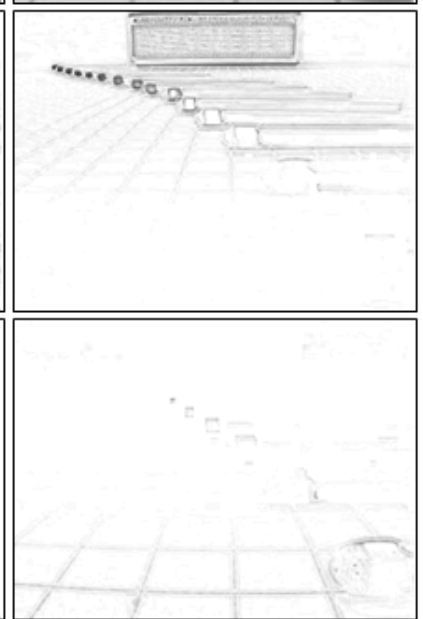

$\mathrm{c}$

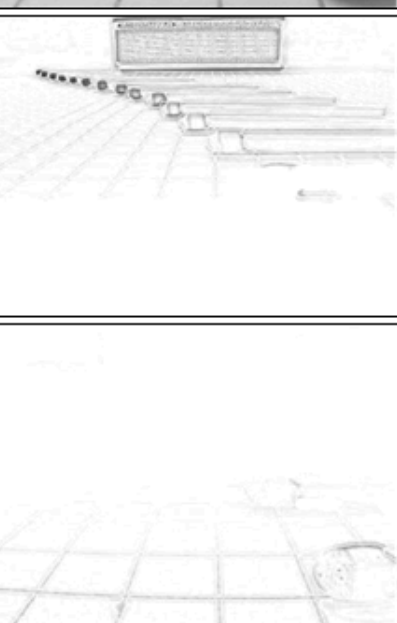

d

Fig. 3. Fused and difference images of the Matches image set: (a) DWT (b) DCT (c) PB (d) Proposed method.

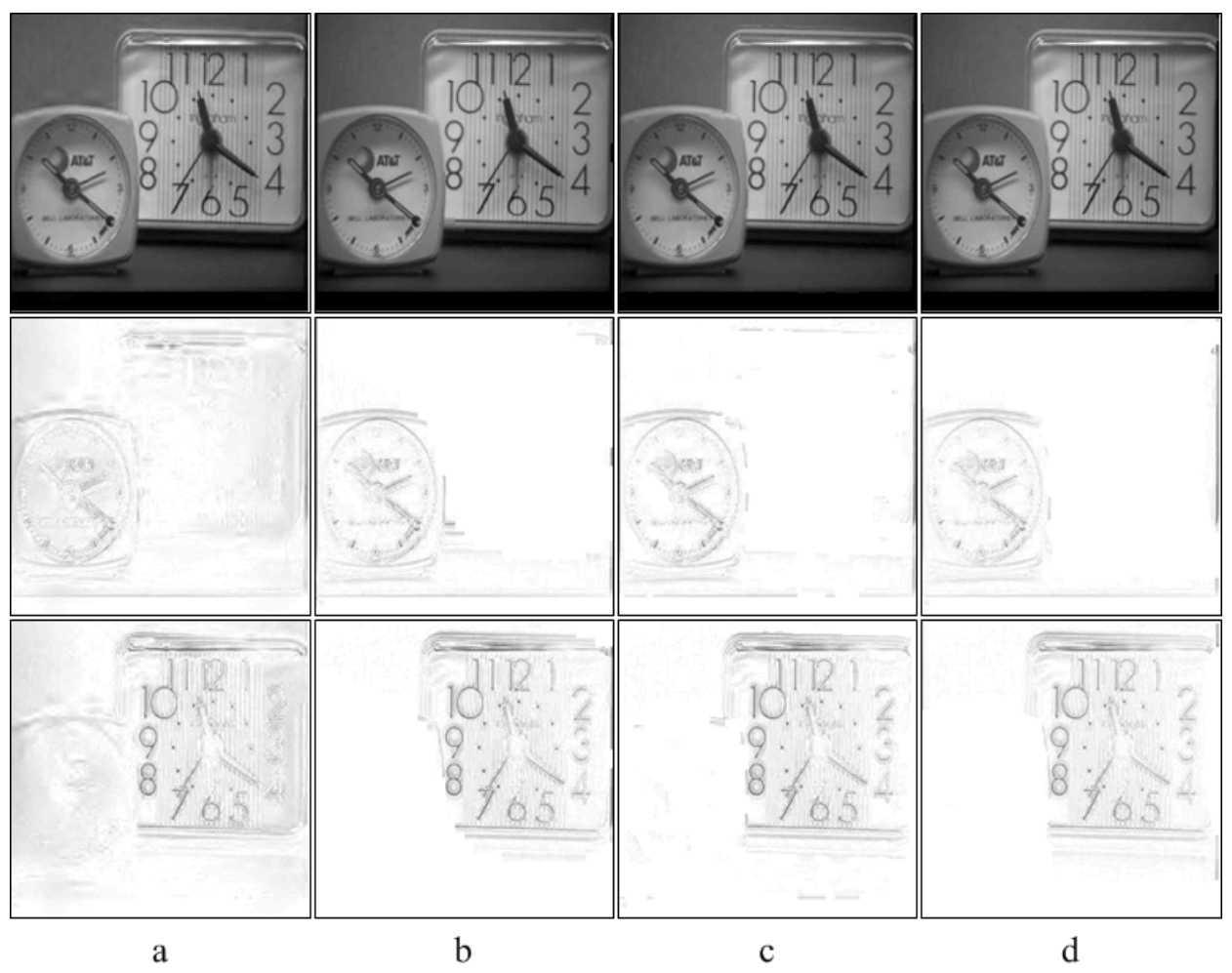

Fig. 4. Fused and difference images of the Clock image set: (a) DWT (b) DCT (c) PB (d) Proposed method 


\section{ACKNOWLEDGMENT}

This paper is supported by Research Foundation of the Erciyes University, Kayseri, Turkey (Grant no: FDK-20155630).

\section{REFERENCES}

[1] V. Aslantas and A. N. Toprak, "A pixel based multifocus image fusion method," Optics Communications, vol. 332, pp. 350-358, 2014.

[2] V. Aslantas, E. Bendes, R. Kurban, and A. N. Toprak, "New optimised region-based multi-scale image fusion method for thermal and visible images," Image Processing, IET, vol. 8, pp. 289-299, 2014.

[3] P. J. Burt and R. J. Kolczynski, "Enhanced image capture through fusion," in Computer Vision, 1993. Proceedings., Fourth International Conference on, 1993, pp. 173-182.

[4] G. Pajares and J. M. de la Cruz, "A wavelet-based image fusion tutorial," Pattern Recognition, vol. 37, pp. 1855-1872, Sep 2004.

[5] M. B. A. Haghighat, A. Aghagolzadeh, and $\mathrm{H}$. Seyedarabi, "Multi-focus image fusion for visual sensor networks in DCT domain," Computers \& Electrical Engineering, vol. 37, pp. 789-797, 2011.

[6] S. Li, X. Kang, and J. Hu, "Image Fusion With Guided Filtering," Image Processing, IEEE Transactions on, vol. 22, pp. 2864-2875, 2013.

[7] S. Li, J. T. Kwok, and Y. Wang, "Combination of images with diverse focuses using the spatial frequency," Information Fusion, vol. 2, pp. 169-176, 2001.
[8] V. Aslantas and R. Kurban, "Fusion of multi-focus images using differential evolution algorithm," Expert System Applications, vol. 37, pp. 8861-8870, Dec 2010.

[9] S. Li and B. Yang, "Multifocus image fusion using region segmentation and spatial frequency," Image and Vision Computing, vol. 26, pp. 971-979, 2008.

[10] B. Yang and S. Li, "Multi-focus image fusion based on spatial frequency and morphological operators," Chinese Optics Letters, vol. 5, pp. 452-453, 2007.

[11] V. Aslantas, "A depth estimation algorithm with a single image," Optics Express, vol. 15, pp. 5024-5029, 2007.

[12] D. Karaboga, B. Gorkemli, C. Ozturk, and N. Karaboga, "A comprehensive survey: Artificial bee colony (ABC) algorithm and applications," Artificial Intelligence Review, vol. 42, pp. 21-57, 2014.

[13] D. Karaboga and B. Basturk, "A powerful and efficient algorithm for numerical function optimization: artificial bee colony (ABC) algorithm," Journal of Global Optimization, vol. 39, pp. 459-471, 2007/11/01 2007.

[14] M. Subbarao, T. C. Wei, and G. Surya, "Focused image recovery from two defocused images recorded with different camera settings," Image Processing, IEEE Transactions on, vol. 4, pp. 1613-1628, 1995.

[15] C. S. Xydeas and V. Petrovic, "Objective image fusion performance measure," Electronics Letters, vol. 36, pp. 308-309, Feb 2000.

[16] Q. Guihong, Z. Dali, and Y. Pingfan, "Information measure for performance of image fusion," Electronics Letters, vol. 38, pp. 313-315, 2002.

[17] C. Yang, J.-Q. Zhang, X.-R. Wang, and X. Liu, "A novel similarity based quality metric for image fusion," Information Fusion, vol. 9, pp. 156-160, 2008. 